\title{
PREPARATION OF LUMINESCENT SILICON NANOPARTICLES BY PHOTOTHERMAL AEROSOL SYNTHESIS FOLLOWED BY ACID ETCHING
}

\author{
X. LI, Y. HE, S.S. TALUKDAR and M.T. SWIHART** \\ Department of Chemical Engineering, University at Buffalo (SUNY), \\ Buffalo, NY 14260-4200, USA
}

\begin{abstract}
$\mathrm{CO}_{2}$ laser-induced pyrolysis of silane (photothermal aerosol synthesis) was used to produce Si nanoparticles. Particles with an average diameter as small as $5 \mathrm{~nm}$ were prepared directly from silane in the gas phase. Etching these particles with mixtures of hydrofluoric acid (HF) and nitric acid $\left(\mathrm{HNO}_{3}\right)$ is shown to be an effective method to reduce the size of the particles produced by silane pyrolysis. After etching, silicon particles with controlled visible luminescence at room temperature were produced. The wavelength of maximum photoluminescence (PL) intensity can be controlled from over $780-500 \mathrm{~nm}$ by controlling the etching time and conditions.
\end{abstract}

Keywords: Silicon; Photoluminescence; Aerosol; Nanoparticles

\section{INTRODUCTION}

The possibility of constructing optoelectronic and luminescent display devices and sensors based entirely on silicon has promoted growing interest in the preparation and characterization of light-emitting silicon nanoparticles (Cullis et al., 1997). Because the particles' luminescence properties are size dependent, multiple colors can be produced using a single material. These particles also have potential applications as fluorescent tags for biological imaging. They can be brighter and much more stable to photobleaching than most of the organic dyes used in these applications and they also have much broader excitation spectra, so that multiple-wavelength emission (from particles of different sizes) can be obtained with a single excitation source.

$\mathrm{CO}_{2}$ laser pyrolysis of silane has been shown to be an effective method to produce moderate quantities of silicon nanoparticles. It produces high-purity loosely agglomerated particles with controlled primary particle size and size distribution. Moreover, laser-assisted synthesis is a continuous process that permits reasonable

*Corresponding author. Tel.: (716) 645-2911 x2205. Fax: (716) 645-3822. E-mail: swihart@eng.buffalo.edu 
production rates. A native oxide layer forms around the silicon nanoparticles when they are exposed to air (Borsella et al., 1997; Botti et al., 1998).

In order to show visible photoluminescence (PL), silicon nanoparticles must be smaller than $5 \mathrm{~nm}$, and their surface must be hydrogen terminated or coated with a high-quality oxide layer or other dielectric. The size of silicon nanoparticles can be reduced by etching them in mixtures of hydrofluoric acid (HF) and nitric acid $\left(\mathrm{HNO}_{3}\right)($ Seraphin et al., 1997).

In this contribution, preliminary results on the synthesis and characterization of $\mathrm{Si}$ nanoparticles produced by $\mathrm{CO}_{2}$ laser-driven pyrolysis of silane in a continuous-flow reactor followed by chemical etching are presented. The synthesis procedure is outlined, and the photoluminescence characteristics of these silicon nanoparticles are reported.

\section{EXPERIMENTAL METHODS}

The silicon powders were synthesized by laser-induced heating of silane to temperatures where it dissociates, in the reactor shown schematically in Fig. 1. Silane can absorb the laser energy at $10.6 \mu$. Sulfur hexafluoride $\left(\mathrm{SF}_{6}\right)$ may be added to the precursor stream as a photosensitizer. $\mathrm{SF}_{6}$ has a large absorption cross-section at the laser wavelength of $10.6 \mu$. Helium and hydrogen flows confine the reactant and photosensitizer $\left(\mathrm{SF}_{6}\right)$ to a region near the axis of the reactor and prevent them from accumulating in the

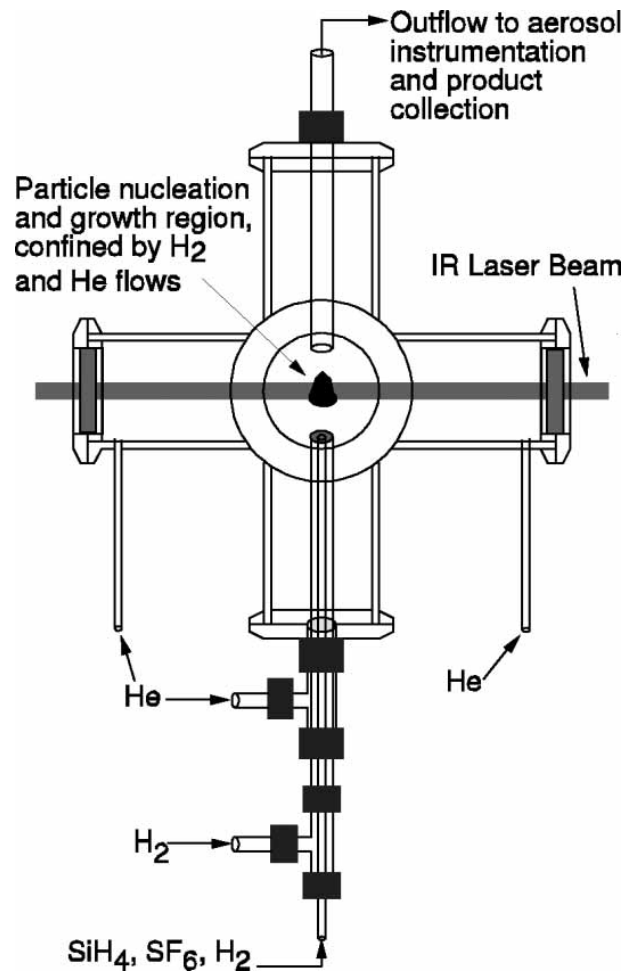

FIGURE 1 Schematic of laser-driven aerosol reactor. 
arms of the 6-way cross from which the reactor is constructed. Hydrogen also serves to increase the temperature at which particle nucleation occurs, and to decrease the particle growth rate, since it is a by-product of the silane dissociation and particle formation.

The powders obtained as described above were dispersed in methanol using mild sonication, then etched with solutions of $0.5-4 \% \mathrm{HF}$ and $20-30 \% \mathrm{HNO}_{3}$ in water to reduce the particle size and passivate the particle surface. Acid solutions were prepared from 49 to $51 \mathrm{wt} \% \mathrm{HF}, 70 \% \mathrm{HNO}_{3}$, and DI water in the necessary proportions. After etching, the particles were collected on polymeric membrane filters and washed with water and methanol.

The silicon nanoparticles were characterized by transmission electron microscopy (TEM) and specific surface area measurements (Brunauer-Emmett-Teller method) prior to etching, and by TEM after etching. Photoluminescence spectra (fluorescence spectra) were recorded with a Perkin-Elmer LS 50 fluorescence spectrometer, with the excitation wavelength set to $355 \mathrm{~nm}$ and a $351 \mathrm{~nm}$ bandpass filter used to suppress any scattered light from the source.

\section{RESULTS AND DISCUSSION}

\section{Characterization of Si Nanoparticles Made by Laser-driven Pyrolysis of Silane}

Selected area electron diffraction patterns obtained from the Si nanoparticles (Fig. 2, inset) show that the particles are either a mixture of crystalline and amorphous material or that the crystallite size is too small to provide a clear diffraction pattern. The TEM micrograph (Fig. 2), along with many others like it, shows that the as-synthesized powder in this case consists of spherical particles, with an average diameter near $5 \mathrm{~nm}$.

Nitrogen physisorption (the BET method) was used to measure the specific surface area of the particles shown above. The measured surface area for this sample was

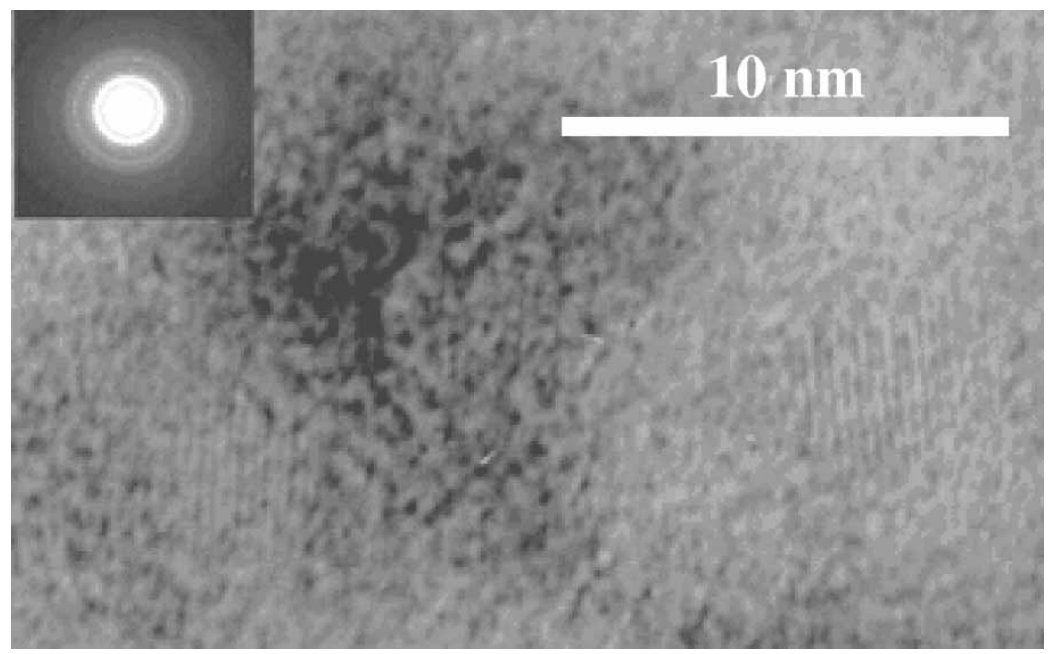

FIGURE 2 TEM image of silicon nanoparticles produced by $\mathrm{CO}_{2}$ laser-driven pyrolysis of silane. The inset shows the selected area electron diffraction pattern from the particles in the image. 
$500 \mathrm{~m}^{2} / \mathrm{g}$. For spherical particles with the same density as bulk silicon, this is equivalent to a particle diameter of $5.2 \mathrm{~nm}$. This is in close agreement with the TEM images. Particle size, crystallinity, and production rate can be controlled by varying the flow rates of $\mathrm{H}_{2}$ and $\mathrm{He}$ to the reactor and by addition of $\mathrm{SF}_{6}$. Changing these parameters allows us to produce particles with average diameters in the range of 5-20 nm. Larger particles are also easily produced, but are not presently of interest. Figure 3 shows examples of the dependence of primary particle size on carrier gas flow rates (with $\mathrm{SiH}_{4}$ flow rate and all other conditions held constant) for typical operating conditions.
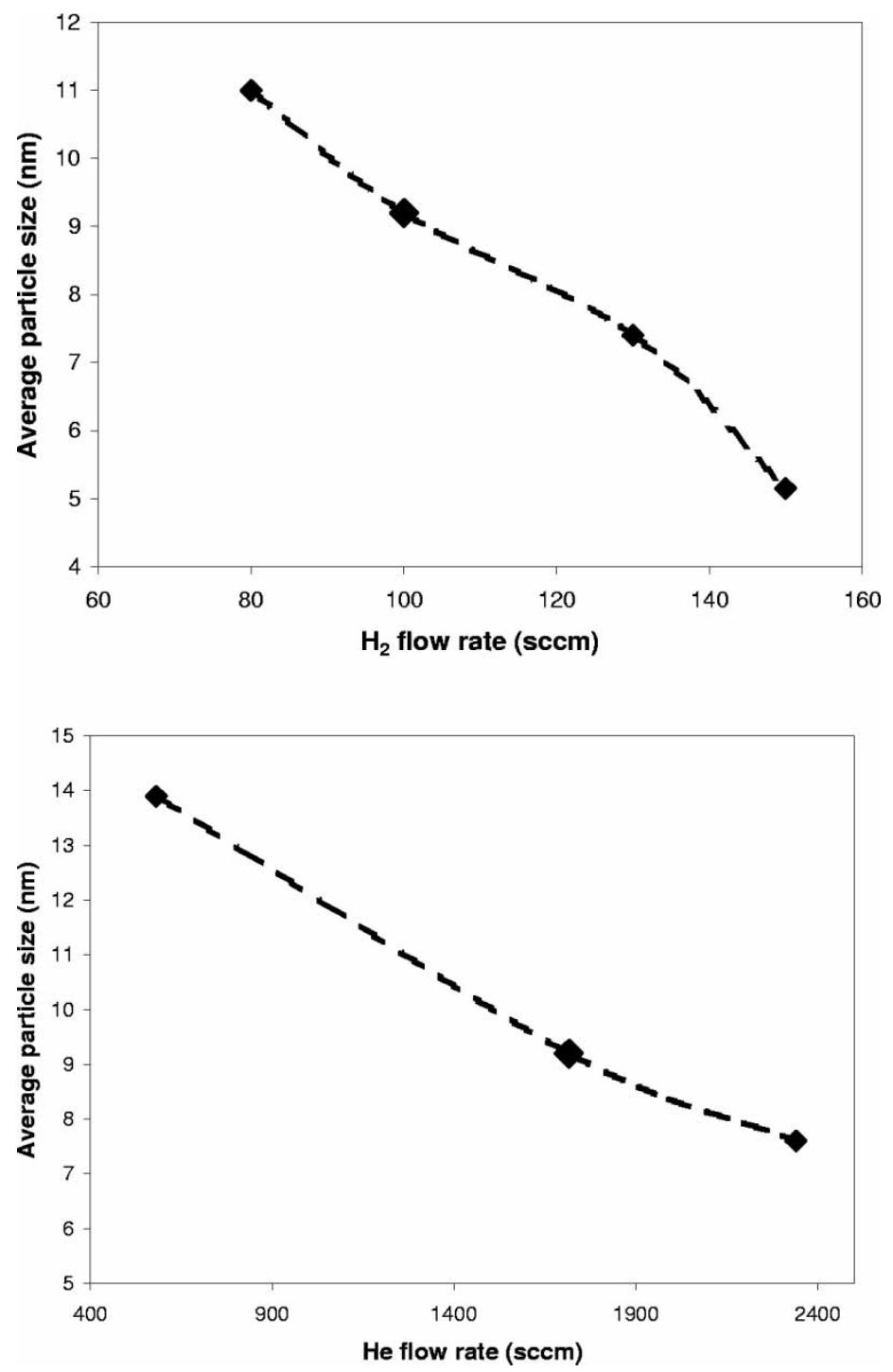

FIGURE 3 Dependence of particle size on carrier gas flow rates for typical reactor conditions. Particle diameter is based on BET surface area measurement. 


\section{Photoluminescence from Etched Silicon Nanoparticles}

Upon initial synthesis in the laser-driven reactor, the silicon nanoparticles exhibit no visible PL either as a dry powder or dispersed in solvents. After etching with $\mathrm{HF} /$ $\mathrm{HNO}_{3}$ mixtures as described above, the particles exhibit bright visible PL. Powder samples and particle dispersions (powders dispersed in water or methanol) with bright visible PL ranging from red to green have been produced, as shown in Figs. 4 and 5. This size-dependent photoluminescence is comparable to that produced by the best published examples of silicon nanoparticles produced by electrochemical etching of silicon wafers e.g., Belomoin et al. (2002).

In Fig. 4, the peak PL emission wavelength decreases from well above 800-562 nm with increasing etching time. In other samples, we have seen peaks in the PL spectrum at wavelengths as short as $506 \mathrm{~nm}$ after etching. For powder samples with peak PL at wavelengths of $600 \mathrm{~nm}$ or longer, the PL intensity and spectrum is quite stable. However, preliminary results suggest that for dispersions of particles in water or methanol and for samples with initial PL peak emission at shorter wavelengths, the spectrum changes with time after the etching procedure. Figure 5 shows the time dependence of the PL spectrum of a dispersion of particles in methanol. Over a period of $125 \mathrm{~h}$, the peak PL intensity dropped by a factor of 2.5 and the peak position shifted by about $12 \mathrm{~nm}$ toward the blue. In other dispersions in methanol, we have observed similar blue shift in the peak position, but with an increase in the PL intensity by a factor of 3. For water dispersions, the changes tend to be even larger. In one sample, the peak position shifted by almost $50 \mathrm{~nm}$ toward the blue and the intensity decreased by almost an order of magnitude during the first $31 \mathrm{~h}$ after preparation. The only powder sample for which we have seen significant change with time was one with an initial peak PL at $506 \mathrm{~nm}$, which moved toward the red by about $40 \mathrm{~nm}$ during the first two days after preparation. These changes can result from slow (ultimately self-limiting) oxidation of the particles, producing a thicker oxide layer and smaller core diameter, or can result from other changes in the state of the particle surface. We are currently investigating surface treatment of the particles to stabilize their PL properties when dispersed in solvents.

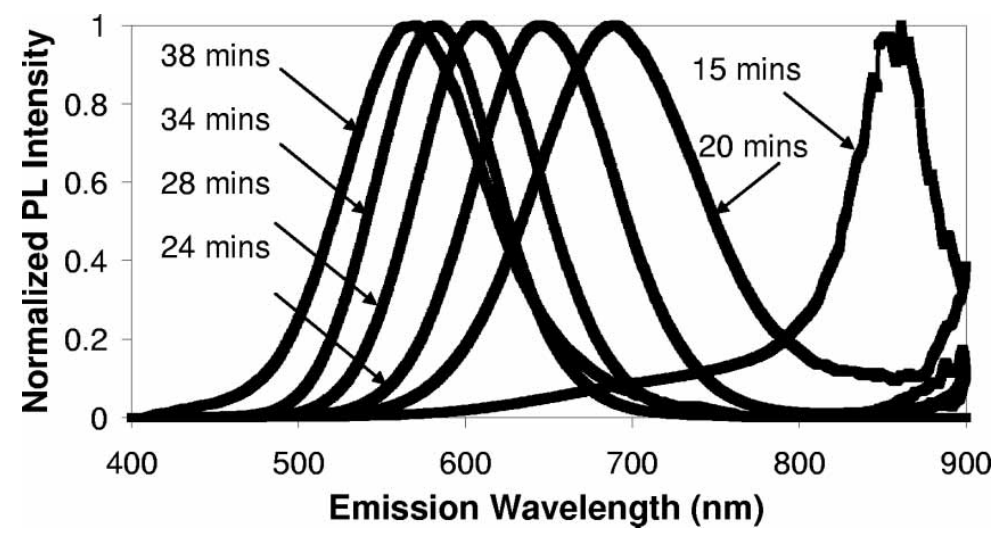

FIGURE 4 Normalized photoluminescence spectra of dry powder samples shown in Fig. 4(a), showing the decrease in peak emission wavelength with increasing etching time (and therefore with decreasing particle size). The sensitivity of the photomultiplier tube used as a detector in these experiments falls off sharply beyond $800 \mathrm{~nm}$, and this is the source of the odd shape of the $15 \mathrm{~min}$ spectrum. 


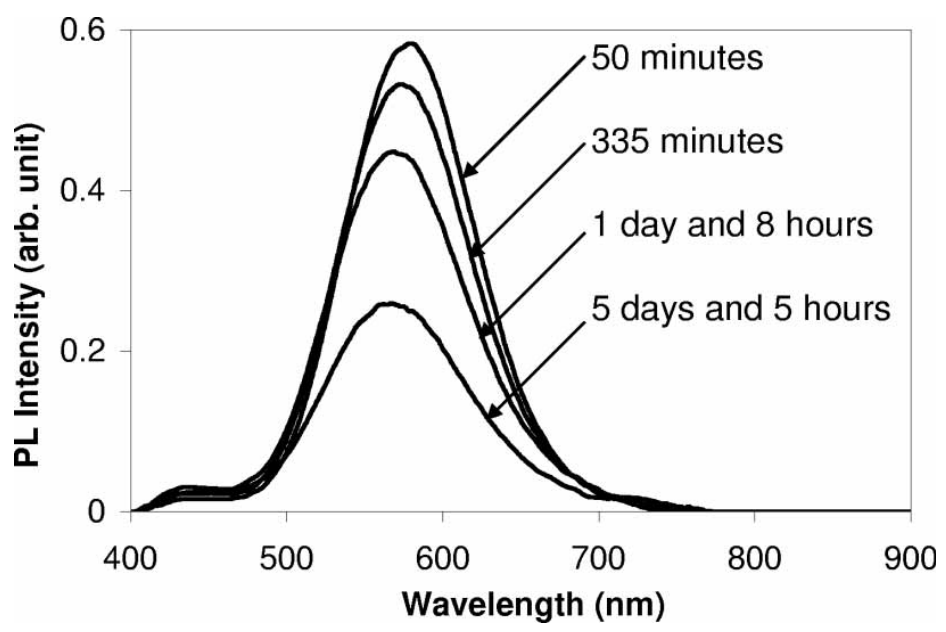

FIGURE 5 Time dependence of PL spectrum from silicon nanoparticles dispersed in methanol.

Furthermore, the dispersions in water and methanol discussed here are not stable colloidal dispersions. After some time, the nanoparticles agglomerate and settle out of these dispersions. It appears that thermodynamically stable dispersions of these particles can be obtained in diols and triols. We are presently functionalizing the particle surfaces to create stable dispersions in a variety of polar and nonpolar solvents. Such surface functionalization can take advantage of known chemistries for attaching molecules to silicon and silica surfaces, so there are many possible functionalization strategies that can be used.

\section{SUMMARY AND CONCLUSIONS}

Silicon nanoparticles with an average diameter of about $5 \mathrm{~nm}$ were prepared by $\mathrm{CO}_{2}$ laser-driven pyrolysis of silane (photothermal aerosol synthesis). After $\mathrm{HF} / \mathrm{HNO}_{3}$ etching, Si particles with controlled visible luminescence were produced. The wavelength of maximum PL intensity can be controlled from above $800-500 \mathrm{~nm}$ by controlling the etching time and conditions. Si nanoparticle dispersions in water and methanol with bright visible PL were also produced and characterized. Dispersions of these Si nanoparticles in both water and methanol exhibited blue shift in their PL spectra with time as well as changes in PL intensity. PL from powder samples was generally stable with time, except for those with very green emission, for which the PL tended to shift toward the red with time.

\section{Acknowledgements}

We thank P.N. Prasad, A.N. Cartwright, W.D. Kirkey and Y. Sahoo for many helpful discussions, Liping Guo for conducting TEM microscopy of the nanoparticles, and Eli Ruckenstein for use of the BET surface area analysis system. 


\section{References}

Belomoin, G., Therrien, J.A.S. et al. (2002). Observation of a magic discrete family of ultrabright Si nanoparticles. Appl. Phys. Lett., 80, 841.

Borsella, E., Botti, S.M.C. et al. (1997). Photoluminescence from oxidized Si nanoparticles produced by CW $\mathrm{CO}_{2}$ laser synthesis in a continuous-flow reactor. J. Mater. Sci. Lett., 16, 221.

Botti, S., Celeste, A. and Coppola, R. (1998). Particle size control and optical properties of laser-synthesized silicon nanopowders. Appl. Organometallic Chem., 12, 361.

Cullis, A.G., Canham, L.T. and Calcott, P.D.J. (1997). The structural and luminescence properties of porous silicon, J. App. Phys., 82, 909.

Seraphin, A.A., Werwa, E. and Kolenbrander, K.D. (1997). Influence of nanostructure size on the luminescence behavior of silicon nanoparticles thin film. J. Mater. Res., 12, 3386. 